\title{
The Impact of Simulated Kraft Substrates on Consumer Attention at the Point of Sale
}

\author{
Rupert Andrew Hurley \\ Clemson University \\ ruperth@g.clemson.edu \\ Julie C. Rice \\ Clemson University \\ jrice3@g.clemson.edu
}

\author{
Gabrielle T. Conlon \\ Clemson University \\ gconlon@g.g.clemson.edu
}

\author{
Charles E. Tonkin \\ Clemson University \\ tonkin@,clemson.edu
}

\author{
Liam O'Hara \\ Clemson University \\ lohara@clemson.edu
}

\begin{abstract}
In order to remain on store shelves and stay competitive among the overwhelming number of packaged goods on today's shelves, companies continue to cut packaging material and increase sustainability. Current packaging has made use of a variety of paperboard materials including Solid Bleached Sulfate (SBS), Coated Recycled Board (CRB) and Uncoated Recycled Board (URB), also known as Kraft. While both SBS and CRB feature a smooth, white printing surface ideal for high quality graphics, Kraft is typically associated with a dull printing surface and lower-quality graphics.

Companies and brands interested in marketing to the eco-friendly consumer are printing a simulated Kraft look on SBS and CRB board rather than utilizing a natural Kraft substrate. The packages printed on natural Kraft substrate (URB) or simulated Kraft substrate (CRB) may or may not affect attention of the consumer when shopping. This research sought to investigate this by using eye-tracking metrics collected from participants in a retail-shopping environment. Statistical analysis yielded no significant difference for participant's attention when shopping for packages made from (URB) or (CRB). This study illustrates how consumer attention can factor into purchasing products packaged with Kraft substrate compared to products packed with simulated Kraft substrate.
\end{abstract}

Key Words: Sustainability, Eye-tacking, Profiling, Package design, CUShop ${ }^{T M}$ 


\subsection{INTRODUCTION}

The consumer shopping experience has been transformed from a rational experience into an emotional one. Due in part to the overwhelming amount of products on grocery store shelves, coupled with the sheer size of today's mega-stores, consumers lack the "mental bandwidth" and time to logically compare all of their options [1].

Packaging can be broken down into two main components: graphical and structural. This research explores the relationship of the main structural component of a package and how it is often used as a simulated graphical element. Methodology was established to test the elements of a natural Kraft material vs. a printed or simulated natural Kraft material on a bleached surface. These elements were evaluated using eye-tracking technology in a retail environment. This technology has recently grown across new markets and can be tested on subjects using a monitor or with glasses in a controlled environment. Marketers use eyetracking software to capture the eye movement of subjects. The captured eye-movement data can be used to determine the influence of a package design placed on a shelf [2]. Ultimately, the goal of the research is to determine if consumers have a difference in attention between packages made with Kraft or simulated Kraft to aid marketers and packaging designers when choosing a proper substrate.

\subsection{BACKGROUND}

With more than two-thirds of consumer purchase decisions made at the point of sale, marketers must find a way to distinguish their packaging and retail presentation at store shelf. Because of this, consumer product companies are consistently looking for ways to increase the shelf presence of their products [3]. Various aspects of a package are used to differentiate from other brands, creative visibility on the shelf and support are shoppers' primary concerns for protecting and promoting the product. Certain packaging aspects are utilized to achieve superior quality at the shelf such as branding, graphics, color, material, and shape. Previous studies have been done to show how food and package appearance can influence a consumer's experience with the product [4].

Along with these aspects, some marketers choose to focus on packaging sustainability as well to attract the consumer [5]. Establishing one set of guidelines for assessing packages from a sustainable marketing or sustainable packaging standpoint will be a complicated, lengthy and daunting task. As previous research shows, consumers may not necessarily understand sustainability with regards to packaging, and an environmental factor may not necessarily increase purchase intent [6]. Similarly, a research study conducted by Perception Research Studies (PRS) in 2008 was completed to quantify consumers' understanding and perception surrounding sustainable packaging [7]. This study was conducted by in-person interviews of a minimum of 100 shoppers from four global markets, US, UK, Germany, and China. The interviews prompted subjects to touch and hold physical unbranded packages and answer specific questions about the packaging systems, materials, and environmental factors, and also general questions about packaging and the environment to understand the subject's attitudes and perceptions across categories [7]. The findings of the study show a lack of understanding of the term "sustainable packaging" from consumers from all four countries. Only $15 \%$ of consumers in the UK, $16 \%$ in the US, $19 \%$ in Germany, and 35 $\%$ in China, claimed to know what the term meant. While sustainability may be a driving factor for a 
manufacturer, consumers do not always recognize it because they simply may not know enough about the package's environmental factors [5].

However, many consumer product companies and packaging designers see an advantage to sustainable packaging on the shelf. A retail audit of today's grocery store shelves will display packages that are utilizing sustainable materials and also packages that appear to be made of sustainable materials. A variety of companies and brands are moving towards a sustainable packaging feel by using a natural looking paperboard, or uncoated recycled board (URB), also known as Kraft board. However, many packages that appear to be made of Kraft board are actually simulated during the printing process. This simulated look is typically printed on either coated recycled board (CRB), also known as clay-coat, or solid bleached sulfate (SBS), two popular food grade paperboards used to package many consumer product goods.

Companies may be hesitant to print on an uncoated board for a few reasons, including lower print quality, line changeover, and perceived quality by the consumer. As manufacturers like Sonoco Products Company continue to develop advanced paperboard, similar to EcoTectTM, most of these causes for concern can be eliminated. For this type of study, eye tracking allows marketers to pretest the shelf impact of packages, products, or point-of sale systems before introducing them at full-scale (Young 2000a). Its availability has recently grown across new markets and can be tested on subjects using a monitor or with glasses in a controlled environment. Marketers use eyetracking software to capture the eye movement of subjects.

\subsection{MATERIAL AND METHODS}

\subsection{STIMULI}

Fifty-two voluntary participants shopped for three consumer products typically packaged with paperboard: cookies, cereal, and pasta. The stimuli were designed to reflect designs of current cereal, cookie, and pasta packaging while remaining brand-generic. The stimuli each contain generic fake brands in order to eliminate brand-loyalty bias. Each stimulus required two designs: one control and one experimental. The goal of the study required the designs to be as identical as possible. Design 1 for each stimulus was printed on the clay-coat (CRB) and Design 2 was printed on Kraft (URB). In order to simulate a Kraft look on Design 1, a sample of the Kraft substrate used was scanned using an Epson scanner. The scanned image was used as a separate layer behind all graphical elements printed on the clay-coat board. All prototypes were printed on a Roland Vera UV inkjet printer and cut and creased on a Kongsberg XL MultiCUT finishing table with Esko iCUT software.

\subsection{PROFILE CREATION}

In order to achieve production quality samples for both substrates, a profile was created for each substrate specific to the printer used for sample creation (Roland Versa UV). For the claycoat, an Esko IT8.7/3, EyeOne IO chart was printed with the exact settings that would be used to print the final prototypes. For the Kraft substrate, the EyeOne Profiling chart was printed three times: with two layers of white ink, with one layer of white ink, and with no white ink.

A production quality sample printed on Kraft substrate would have a minimum of one and a maximum of two layers of white depending on the number of ink stations on the printer. 

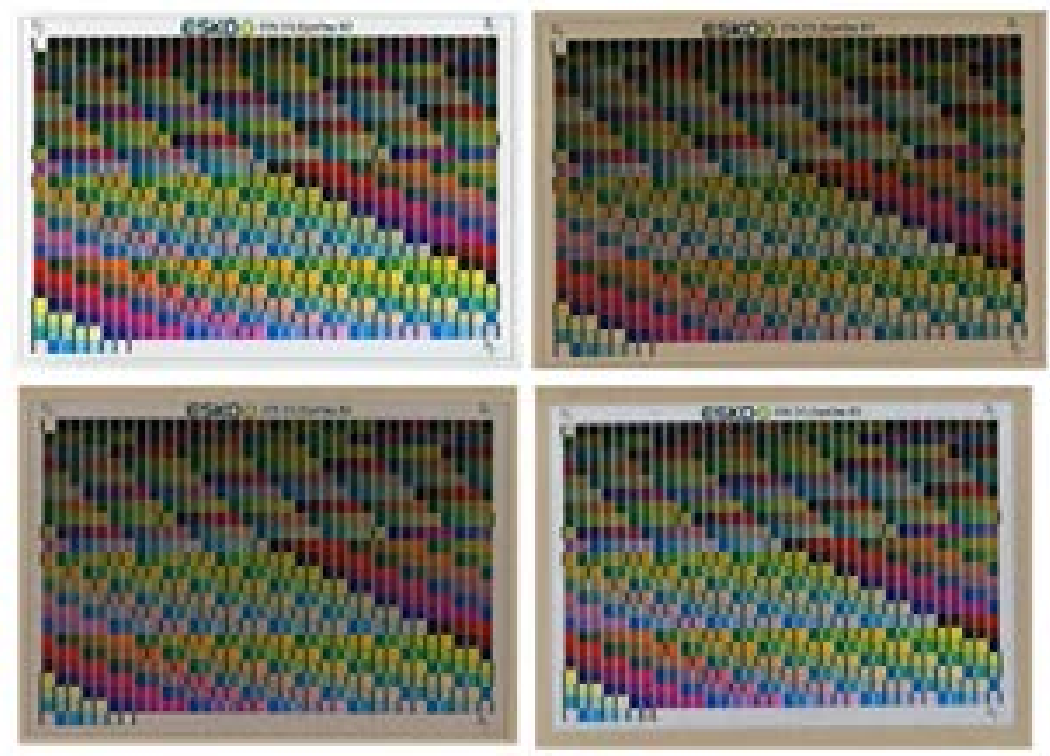

Figure 1: Printed profile charts: Clay-coat (top left), Kraft (top right), Kraft with one layer of white ink (bottom left), Kraft with two layers of white ink (bottom right)

This would be determined based on the needs of the customer. In this case, both options were tested because they had such similar E00 values when compared to the flexographic sample with one layer of white ink. A sample with one layer of white ink, two layers of white ink, and with no white ink was printed to determine visually and scientifically which sample created the smallest $\mathrm{E}$ value.

The charts printed on Kraft visually show dullness in color when compared to the claycoat (Figure 1). The profiles are created to ensure individually each prototype is printe

Each printed profile chart was measured against the control using an ilIO eXrite spectrophotometer and Color Engine Pilot 12.1 at the Sonoco Institute Esko Lab. The spectrophotometer takes ten $\mathrm{L} * \mathrm{a} * \mathrm{~b} *$ measurements for each square and averages them (after throwing out the first and last measurement).
It records average $\mathrm{L}^{*} \mathrm{a} * \mathrm{~b} *$ values to compare to the intended $\mathrm{L}^{*} \mathrm{a}^{*} \mathrm{~b}^{*}$ values on the original chart send to the printer.

Next a profile was created for each substrate using I1Profiler. The profile was created by importing the control chart (Esko IT8.7/3, EyeOne IO) and the measured L*a*b* values from the printed charts. Four total profiles were created, all using the same Esko IT8.7/3 with the EyeOne IO chart as the control.

Also using the Esko I1Profiler, each of the three created profiles for Kraft (no white, 1 layer of white, 2 layers of white) was compared to the profiles for clay-coat. Using the compare tool, the software averages the $\mathrm{E}$ of the $\mathrm{L}^{*} \mathrm{a} * \mathrm{~b}^{*}$ values (Table 1, pg. 29). From this data, it was determined the Kraft prototypes should be created with two layers of white because it has the smallest average E when compared to the clay-coat. 


\begin{tabular}{|l|l|l|l|}
\hline \multicolumn{3}{|l|}{ Profile Comparison Data $\left(\mathbf{D 5 0 - 2}^{\circ}\right)$} \\
\hline Profile 1 & Profile 2 & Average $\Delta \mathbf{E}_{\mathbf{0 0}}$ & Standard Deviation \\
\hline Clay-coat & Kraft & 13.58 & 6.15 \\
\hline Clay-coat & Kraft + 1 Layer of White Ink & 10.16 & 5.23 \\
\hline Clay-coat & Kraft + 2 Layers of White Ink & 4.19 & 2.30 \\
\hline
\end{tabular}

Table 1. Average E values for Kraft profiles compared to clay-coat profile

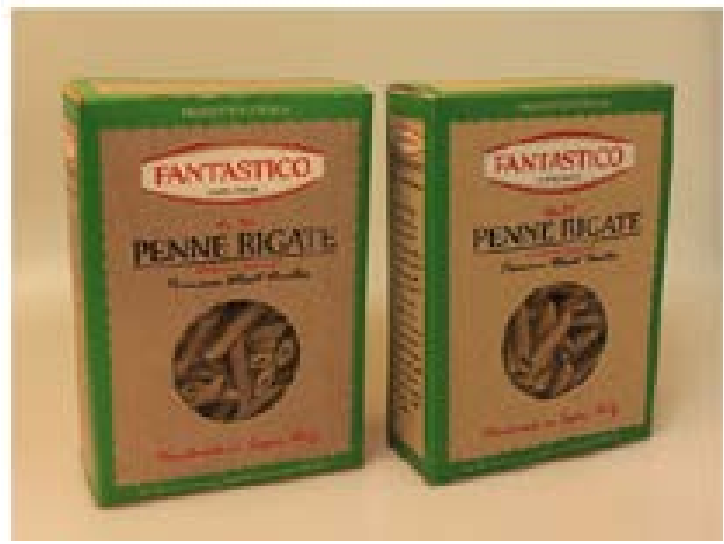

Figure 2: Final Clay-coat Pasta Stimuli (left) and Kraft Pasta Stimuli (right)

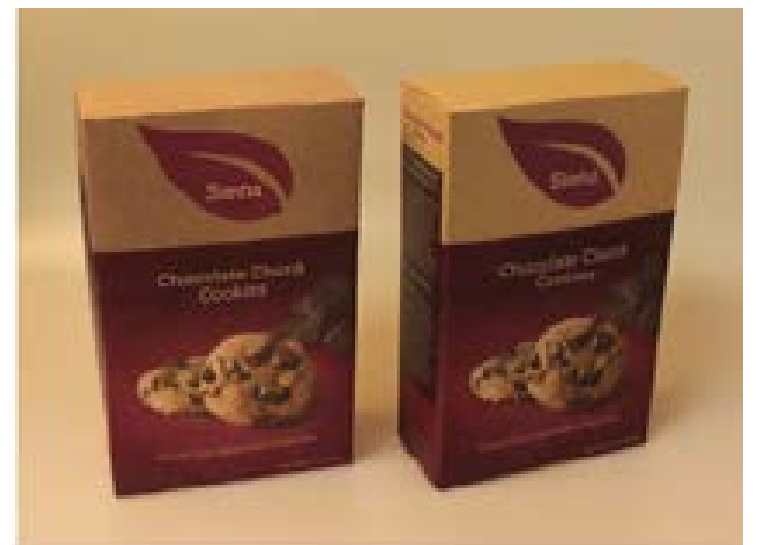

Figure 3: Final Clay-coat Cookie Stimuli (left) and Kraft Cookie Stimuli (right)

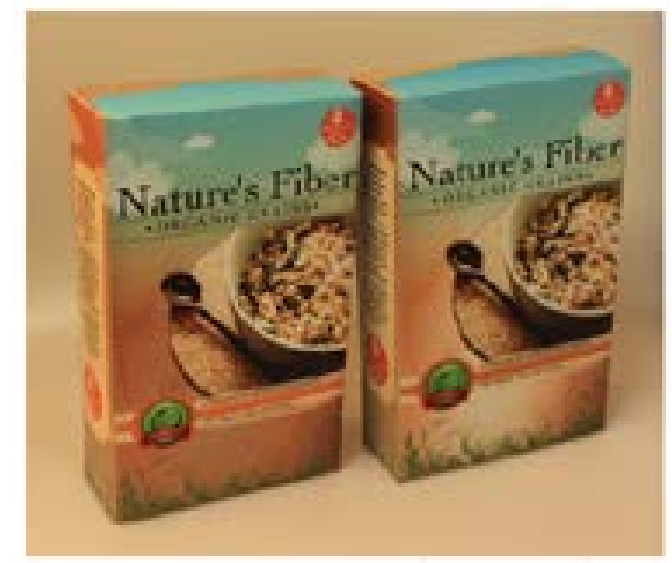

Figure 4: Final Clay-coat Cereal Stimuli (left) and Kraft Cereal Stimuli (right)

Profiles were applied to print files using a custom design workflow in Esko Automation Engine Pilot. Final PDF files were ripped to the print software for final production. Four total stimuli were created for each product category: pasta, cookies, and cereal (Figures 2-4). Two control stimuli samples were printed on clay-coat and two experimental samples were printed on Kraft. 


\subsection{APPARATUS}

The Tobii Glasses Eye Tracking system consists of eye tracking glasses, a recording assistant, IR markers, and Tobii Studio eye tracking software. The eye tracking glasses are equipped to follow eye pupil movements following calibration. The recording assistant is hardwired to the glasses and acts as a control interface. It is used for individual calibration and records tracking and visual data using a standard transferrable secure digital (SD) memory card. IR markers have a transmission range of $60-250 \mathrm{~cm}$ at angles between $90^{\circ}$ and $150^{\circ}$. IR markers are positioned around the packages being tested, known as the visual area of interest or AOA. Four or more markers are used in conjunction to form a plane in which Areas of Interest or AOI's are positioned based on the subject's eye movements. Eye tracking data is transferred to the Tobii Studio eye tracking software for analysis.

\subsection{EXPERIMENTAL DESIGN}

Products were arranged on the shelves similar to how they would appear in a grocery store. The pastas stimuli was placed with other pasta packages, the cereal stimuli was placed with other cereal packages, and the cookie stimuli was placed among other cookie packages. All stimuli were placed at eye level to improve quality of eye tracking.

Areas of Analysis (AOA's) and Areas of Interest (AOI) are mapped on each figure. AOA's are determined by the location of a grid of IR markers on the store shelves. This is the area where eye-tracking data is recorded for each participant. Inside the AOA is an AOI, Area of Interest, specifically mapped for each stimulus. Eye-tracking data will be compared for the AOI for control stimuli vs. experimental stimuli. Control stimuli were replaced with experimental stimuli halfway through the study to ensure equal participant group sizes.

Each participant was provided a shopping list and instructed to shop for items as they would normally shop. Shopping lists were randomized to ensure participants were not influenced by the order in which they shopped. Each stimulus (cookies, pasta, and cereal) was included to ensure participants shopped for these specific items. The other three items were chosen randomly and positioned strategically in CUShop ${ }^{\mathrm{TM}}$ so that participants shopped throughout the entire store.

\subsection{PROCEDURE}

Voluntary participants were informed of the nature of the study as a consumer-packaging, eyetracking study. Participants were required to read over an informed consent form notifying them there were no known risks involved and that they could choose to stop participating at any time. Participants were informed that the study would last approximately 15 minutes and were asked to complete a 3-step process to complete the study.

Each participant began by placing the eyetracking glasses on their eyes and looking towards a blank wall. A trained researcher instructed them to follow an IR-marker with their eyes to calibrate the camera on the glasses.

The participants were handed a shopping list attached to a clipboard and pen and were asked to shop as they would normally shop. They were instructed to write the corresponding number for the product chosen in boxes provided on the shopping list. Participants were asked to exit CUShop ${ }^{\mathrm{TM}}$ upon completion of the shopping task. Following the shopping experience, participants were asked to complete a short survey on a computer to gain 
insight into the consumer experience and collect demographic and background information. They were led to a small room with a computer where the survey was taken in private. Data was recorded using the survey software SurveyMonkey.com.

\subsection{DATA COLLECTION AND EYE- TRACKING METRICS}

Using Tobii Studio, Areas of Analysis (AOA's) and Areas of Interest (AOI's) were predetermined for each product category (cookies, cereal and pasta). AOI's for each product are located within specific AOA's determined by the placement of IR (Infrared) markers on the store shelves. AOI's were used to determine two measurements of participant eye-movement: Time to First Fixation (TTFF) and Total Fixation Duration (TFD). TTFF is defined as the time it takes for a participant to fixate on an AOI. TFD is the total time that a participant looks at one particular AOI.

The survey questions were written to gain insight into the participants' normal shopping behaviors and decisions as well as demographic information. The data stored through SurveyMonkey.com was exported in Excel format after the completion of the two-day experiment.

Statistical anaylysis was run on the raw eye tracking data collected using Tobii Studio. A Wilcoxon Rank Sum test was used to determine if the measured data was significantly different for the Clay-coat stimuli vs. the Kraft stimuli.

\subsection{RESULTS AND DISCUSSION}

\subsection{SURVEY FINDINGS}

After exiting CUShop ${ }^{\mathrm{TM}}$ upon completion of the eye-tracking portion of the study, each participant was asked to complete a follow-up survey located in a room adjacent to the CUShop ${ }^{\mathrm{TM}}$. Each participant was assigned a unique participant number for the duration of the study to ensure confidentiality

Participants' ages ranged from 18-59, with the majority of participants being between the ages of $21-29.86 \%$ of the participants were under the age of 30 due to the location of the study, being at the Sonoco Institute at Clemson University. One participant chose not to report his or her age. Approximately $62 \%$ (or 32 participants) of the sample was male and $38 \%$ (or 20 participants) of the sample was female.

Fifty percent of participants reported as the primary shopper for their household with an additional $33 \%$ of participants who were sometimes the primary shopper. Only $17 \%$ of participants were not the primary shopper for their household. In addition, 53\% of the sample shopped for household items once a week or more, $39 \%$ shopped once every two weeks, $2 \%$ shopped once a month, and about $6 \%$ shopped less than once a month.

Within the last 30 days, $75 \%$ of participants shopped for pasta, $63 \%$ of participants shopped for cereal, and $48 \%$ of participants shopped for sweet snacks, which verifies that the pasta, cereal and cookie stimuli participants were instructed to shop for were typical of their normal shopping experience.

The majority of participants (54\%) responded "somewhat important" when asked if packaging material is an important part of their purchasing decision, while an additional 35\% responded with "very important." Fourty-eight percent of participants responded with "very important" when asked if packaging art (i.e. graphics, design, and 
colors) was an important part of purchase decision. An additional $42 \%$ responded with "somewhat important." Only five participants answered either "neither important nor unimportant," "somewhat unimportant," or "very unimportant." All but one (98\%) participant responded that price was an influential factor when purchasing a new product. The responses ranked in order from most to least are: Price (98\%), Package Design (77\%), Color (60\%), Graphics (58\%), Print Quality (38\%), Material (37\%), and Recyclability (29\%). Four participants chose to answer the "Other" option with their responses including ingredients, product visibility, shelf location, coupons, and product quality.

\subsection{EYE-TRACKING RESULTS AND STATISTICAL ANALYSIS}

The raw eye tracking data collected using Tobii and analyzed using IBM SPSS (Service Product for Statistical Solution). SPSS output was used to determine the mean and standard deviation and standard error for participants TTFF and TFD for each stimulus. Each data set was tested for normality using the Shapiro-Wilk test. After concluding that all data sets are nonnormal, the Wilcoxon Rank Sum test was completed as the non-parametric equivalent to a twosample t-test.

With p-values ranging from 0.367 to 0.925 , there was no significant difference $(\mathrm{p}>0.05)$ found between the TTFF or TFD for stimuli printed on Kraft and stimuli printed on Clay-coat for the cookie, pasta, or cereal stimuli (Table 2).
Participants did not take significantly more time to fixate on the control or the stimuli packages, nor did not spend significantly more time looking at the control or the stimuli packages.

\subsection{CONCLUSION}

The goal of the research was to investigate consumer's attention when purchasing products packaged with Kraft substrate compared to products packed with simulated Kraft substrate. Previous research suggests that consumers generally only prefer "sustainable packaging" when prompted and otherwise do not consider it a factor when making a purchase decision.

Analyzing eye-tracking data from 52 voluntary subjects, participants shopping for all three stimuli, cookies, pasta and cereal, did not take significantly more or less time to fixate on the Kraft packages or the simulated Kraft packages ( $p$-value> 0.05). Also, participants did not spend significantly more time or less time looking at the Kraft packages or the simulated Kraft packages ( $p$-value $>0.05$ ), so there was no difference in consumer attention when shopping for the Kraft packages or the simulated Kraft packages.

\subsection{LIMITATIONS AND IMPLICATIONS FOR FUTURE RESEARCH}

A potential explanation as to why there was no difference between the two stimuli could be due to the absence of physical interaction with the packages. Due to the nature of the eye-tracking glasses, participants were prompted to shop for items

\begin{tabular}{|l|l|l|}
\hline Stimuli & TTFF & TFD \\
\hline Cookie & 0.477 & 0.925 \\
\hline Pasta & 0.875 & 0.798 \\
\hline Cereal & 0.367 & 0.576 \\
\hline
\end{tabular}

Table 2. p-values for stimuli printed on Kraft and stimuli printed on Clay-coat 
without touching the packages or taking them off the shelves. While the simulated Kraft substrate may look very similar to a natural Kraft board, it does not replicate the tactile quality of the natural, uncoated board, which may have an effect on final purchase decision. Another explanation as to why there was no difference between the two stimuli could be due to the small sample size ( $<30$ for each stimuli). In comparing the Clay-coat and Kraft treatments, a larger sample size would have increased the chance of finding a significant difference between the two.

Although the visual differences in the two tested cereal stimuli did not prove to be a factor that influenced participants' TTFF or TFD, it should be noted that in order to use graphics with gradients, the inclusion of white ink should be applied using a different methodology than defined to ensure samples are of equal quality. It is recommended for a new methodology to be developed and the cereal stimuli retested in a similar shopping environment.

It is also recommended to complete a second part of the study to understand purchase decision. The study would have two portions, an eye-tracking portion and an interview portion. The eyetracking portion should test the stimuli in a side-by side manner to determine if there is a preference in Kraft and simulated Kraft packages based on visual shopping alone. The interview portion should test shoppers in the same environment without eye tracking glasses, allowing consumers to make a preference decision by actually choosing items off the shelves that they would purchase. It would be followed up with an interview where participants would be asked about the differences in the Kraft and simulated Kraft packages to gauge knowledge of package materials and typical shopping behaviors. This would allow researchers to make specific conclusions surrounding preference of Kraft and simulated Kraft packages.

\section{REFERENCES}

[1] Young, S. Too Much Choice and the implications for package design. Brand Packaging 2010c, 9-13.

[2] Drew, J., \& Meyer, S. Color Management for Packaging: A Comprehensive Guide for Graphic Designers 2008, 224.

[3] Hurley, R. A., Galvarino, J., Thackston, E., Ouzts, A. and Pham, A. The Effect of Modifying Structure to Display Product Versus Graphical Representation on Packaging. Packag. Technol. Sci. 2012, DOI: 10.1002/pts.1996. Link

[4] Gomes, T, Hurley, R.A, Duchowski, A, Darby, D, Ouzts, A. The Effect of Full BodyVersus Partial Body Graphic Labelling. Packag. Technol. Sci. 2014, DOI: 10.1002/pts.2070. Link

[5] Young, S. Packaging and the environment. Flexible Packaging 2008a, 24-27.

[6] Fitzpatrick, L., James, K., Lewis, H., and K. Sonneveld. Sustainable Packaging: How do we Define and Measure it? 2005, 1-9. Link

[7] Young, S. Packaging and the Environment: A Cross-Cultural Perspective. Design Management Review 2008b, 19(4), 42-48. DOI:10.1111/j.1948-7169.2008.tb00140.x. Link 